\title{
Sailing as Stroke Rehabilitation Strategy
}

\author{
Maximiliano Romero, Giuseppe Andreoni, Silvia Piardi, \\ Andrea Ratti, Beril Imamogullari ${ }^{a}$ and Franco Molteni ${ }^{b}$ \\ ${ }^{a}$ Department of Design \\ Politecnico di Milano \\ Milan, 20158, Italy \\ ${ }^{b}$ Villa Beretta Rehabilitation Centre \\ Valduce Hospital \\ Costamasnaga, 23845, Italy
}

\begin{abstract}
Stroke (brain attack), is a serious global public health problem and the main cause of many forms of disability. The majority of stroke survivors are mostly left with motor (muscle movement or mobility) impairments. Although remarkable developments have been made in drug treatment, post-stroke care continues to rely on rehabilitation interventions mostly. On the other hand, the presence of post-stroke depression has been associated with decreases in functional recovery, social activity and cognition. Therefore, this project aims to improve the quality of people's lives after a stroke by introducing sailing as outdoor mobility rehabilitation. It is intended to increase the patients' motivation and engagement in the rehabilitation process by a more enjoyable and relaxing intervention than the existing ones especially for long term periods. The project, based on a haptic system installed on the deck of NYTEC 28 sailing boat, aim to monitoring rehab process development in post-stroke during normal sailing activity.
\end{abstract}

Keywords: Product Design, Stroke Rehabilitation, Physical Computing, Sailing, User Engagement

\section{INTRODUCTION}

Stroke, also called 'brain attack', is a major public health problem, representing the third leading cause of death and the main cause of disability (Sacco et al, 2011). The risk of stroke is influenced by a number of factors. The most important factors are: previous stroke or transient ischemic attack (a 'mini-stroke'), high blood pressure, physical inactivity, advanced age, diabetes, heart disease and smoking (NIH, 2013).

Each year 15 million people worldwide experience a stroke. About one third of this people with stroke survive with disability (Stroller, 2012). In Italy, the annual incidence of stroke is approximately 130,000 individuals with primary stroke and an additional 50,000 individuals who suffer from recurrent stroke (Fattore, 2012). Furthermore, the prevalence of stroke-related burden is expected to increase over the next two decades (Stroller, 2012).

The majority stroke survivors are mostly left with motor impairments, which restrict function in muscle movement or mobility. Other common impairments include those of speech and language, swallowing, vision, sensation, and cognition (Langhorne, 2011). The most common movement impairment, that affects roughly 85 percent of stroke survivors, is one-sided paralysis, known as hemiplegia, or one-sided weakness, known as hemiparesis. This kind of paralysis/weakness can impact arms, hands, legs and facial muscles (NSA, 2013). Although impressive developments have been made in the medical management of stroke, without a widely applicable or effective medical treatment most post-stroke care will continue to rely on rehabilitation interventions (Langhorne, 2011).

Most common rehabilitation process consists of three main therapies that are physical, occupational, and speech therapy. Physical therapy helps a patient with balance, standing, walking, transitional movements and regaining strength. Occupational therapy helps a stroke victim regain their ability to perform activities of daily living (ADLs) 
such as feeding, grooming, bathing, toileting, and dressing. Occupational therapists also work to improve overall arm function and fine motor skills so survivors are able to perform their ADLs. To experience significant recovery, stroke patients must perform a substantial number of daily exercises at home. Evidence shows that the intensity, frequency of focused therapy and patients' motivation and engagement can improve functional outcomes (Matarić et al., 2007).

On the other hand, stroke can have a devastating effect on people's lives, with half of survivors being dependent on others six months later, one third feeling socially isolated, one quarter having abnormal mood and half not getting out of their house as much as they would like (Logan et al. 2012). Therefore, many post stroke patients lose confidence in their recovery process after not observing any improvements over a long period of time and receive patchy levels of rehabilitation or even discontinue their rehabilitation process. Studies show that only $31 \%$ of longtime patients actually perform their repetitive exercises as recommended by therapists (Alankus et al., 2010).

\section{STATE OF THE ARTS}

\section{Stroke Rehabilitation Process}

Physical and occupational therapy help reverse disabilities caused by stroke. By encouraging the use of the affected parts of the body through exercise, the patient can slowly relearn the ability to use them again. This is a very demanding process that can require hundreds of repeated motions every day to make progress towards recovery (Kwakkel, 1997). Some researches suggests that stroke patients recover lost function in three ways: overcoming learned non-use, learning to use existing redundant neural pathways that do not include damaged brain tissue, and the development of new neural pathways through brain plasticity (Selzer, 2006).

Recovering stroke patients typically participate in some form of therapy program which often consists of the patient performing repeated motions under the supervision of a therapist in a one-on-one session (Langhorne, 2009). Process include active exercises in which the patient repeats one basic motion many times, and purposeful exercises in which the patient carries out simulations of everyday tasks that require a combination of motions. Motion abilities gained are initially hard to control, and require less effort in time. The goal is to get the patients to move their affected limbs as easily as they move their unaffected limbs. Studies have shown that increasing the amount of such exercise results in better recovery (Kwakkel, 1997).

Therapy on the lower extremity is the primary concern in early inpatient stroke therapy in order to enable mobility of the patient. Recovery of the upper extremity has a slower progression and is usually gained through outpatient and home therapy (van der Lee, 1999). Patients with upper extremity paralysis typically regain motion starting from their shoulder. Over time, they may gradually regain motion in the elbow, wrist, and, finally, the hand. Because of limitations on therapy, patients must do much of the work necessary to recover arm function at home, underscoring the need for home- based technologies that can both help and motivate patients to perform therapeutic exercises.

Regarding the maintenance of rehabilitation process after hospital, there is moderate evidence that hospital-based outpatient rehabilitation improves outcomes when compared to routine care over the short-term. However, the benefits are not maintained long-term. In contrast, there is strong evidence that additional home-based rehabilitation does not result in improved functional outcomes when compared to routine care (usually no additional therapy). There is conflicting evidence as to whether hospital-based or home-based outpatient rehabilitation therapies are superior. There is limited evidence that for younger stroke patients, home-based outpatient therapy may improve functional and quality of life outcomes.

Water-based exercises are used in rehabilitation and might help to reduce disability after stroke. The evidence from randomised controlled trials so far does not confirm or refute that water-based exercises might help to reduce disability after stroke. There is a lack of hard evidence for water-based exercises after stroke. Better and larger studies are therefore required. (Mehrholz, 2011).

Water-based exercises are used also in proprioceptive rehabilitation. Rehabilitation programs should be designed to include a proprioceptive component that addresses the following three levels of motor control: spinal reflexes, cognitive programming, and brainstem activity. Proprioception can be defined as a specialized variation of the 
sensory modality of touch that encompasses the sensation of joint movement (kinesthesia) and joint position (joint position sense). Numerous investigators have observed that afferent feedback to the brain and spinal pathways is mediated by skin, articular, and muscle mechanoreceptors. (Lephart, 1997)

Depression is a common complication post-stroke affecting approximately one-third of patients. The presence of post-stroke depression has been associated with decreases in functional recovery, social activity and cognition. In addition, the presence of mental health disorders following stroke may be associated with increased mortality (Salter K. et al 2013).

We can assume that an active daily living, rich of movements, could be good therapy to improve autonomy. On the other hand social activities could help to prevent post-stroke depression. Designing and implementing specific tools and products for improve social and rich of movement activities for post stroke patients could be a product designers role.

\section{Innovative Rehabilitation Design Projects}

To improve patients socialization and engagement, many projects has been conducted at school of design of Politecnico di Milano regarding design for disability in aquatic context. (Piardi et al., 2012) and more specifically regarding stroke rehabilitation. K4YOU is a kayak for encourages sport activity after stroke. Two adjustable outriggers could modify the stability of the kayak and a central pivot help to move the affected arm thanks to the active one (Sessolo, 2014). ATLAS is a device for rehabilitation under the water (Villa, 2014).
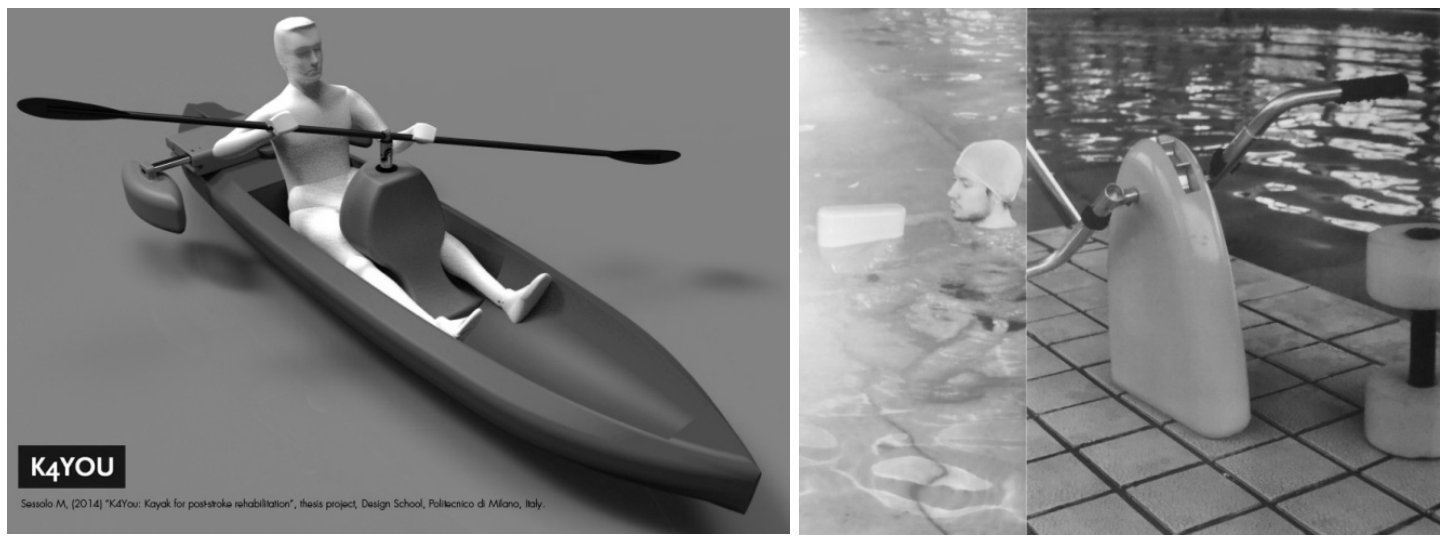

Figure 1 and 2, K4YOU by Matilde Sessolo and Atlas by Andrea Villa, respectively.

Both examples, and majority of considered projects doesn't apply tracking technology or actuators for active and passive rehabilitation. An active tracking rehab system was developed based on Ronny Gardiner Rhythm Music method (Bendit, 2014). The system consists of contact sensors that can tag claps of hands, knee or foot in relation with music. An active music listening experience through an auditory-feedback based Musical Instrument Digital Interface product/system with the goal of providing an accompaniment and guidance to domicile stroke rehabilitation treatment alongside with traditional rehabilitation, that integrate physical, cognitive exercise and entertainment.

Presented project, aim to encourage social activity of post stroke patients in a context of aquatic sports. Sailing, and in particular, school boat was chose as main activity since this context could be rich of personal and team stimulus. Furthermore, sailing is a very frequent hobbies and leisure time in Italy and specifically in Lecco region (where is located one of the campus of Politecnico di Milano).

\section{MATERIALS AND METHODS}

This project is based on three main principles of User Centred Design (Norman 2000). It mean: User involvement, Multidisciplinary of the team and Iterativity of the process. Present paper shown the steps has been performed and the result obtained from methodological point of view. Applied process is composed by five steps which will be performed repetitively as time as necessary to achieve the goal (Figure 3 ). 


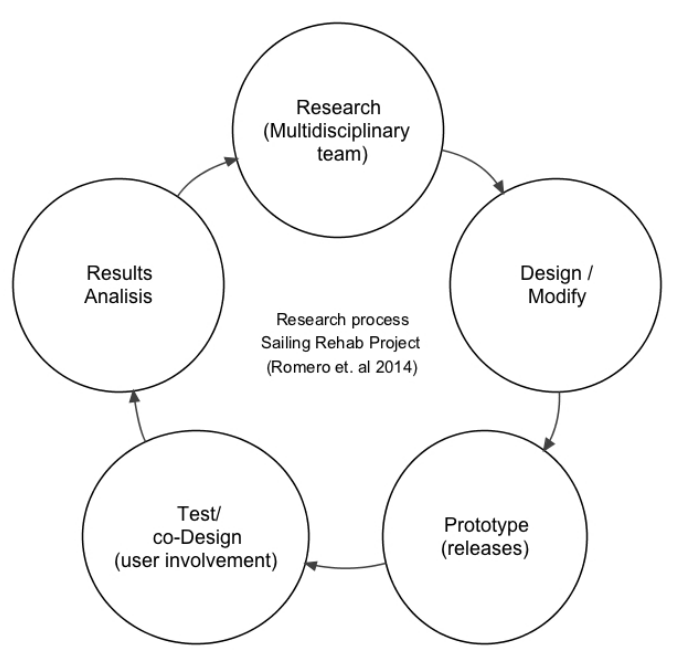

Figure 3. Diagram of research process of iterative cycles.

First cycle of research step, was conduced involving medical expert from Villa Beretta Rehabilitation Centre of Valduce Hospital. An early evaluation of validity of the proposal has been discussed in multidisciplinary meetings. Preliminary design and renders has been produced in order to control technological availability of proposal and sailing specialist has been involved in the project. A training team from Dervio's nautical base of Lega Navale Italiana (Italian Navy League) has been interviewed in order to define the best boat for the project.

\section{The Boat, A Floating Rehab Gymnasium}

According to Lega Navale Italiana and medical team from Villa Beretta Rehabilitation Centre, an Nytec 28 boat has been defined as optimal situation. Nytec 28 is a school boat of 8,5 mt produced by an Italian company since 90 's. The boat can transport 5 or 6 passengers which is an optimal condition to rehabilitative activities. 1 skipper, 1 therapist and 3 patients in rehab process. The boat could have great stability during navigation, which is particularly useful for the first steps in proprioceptive rehabilitation.
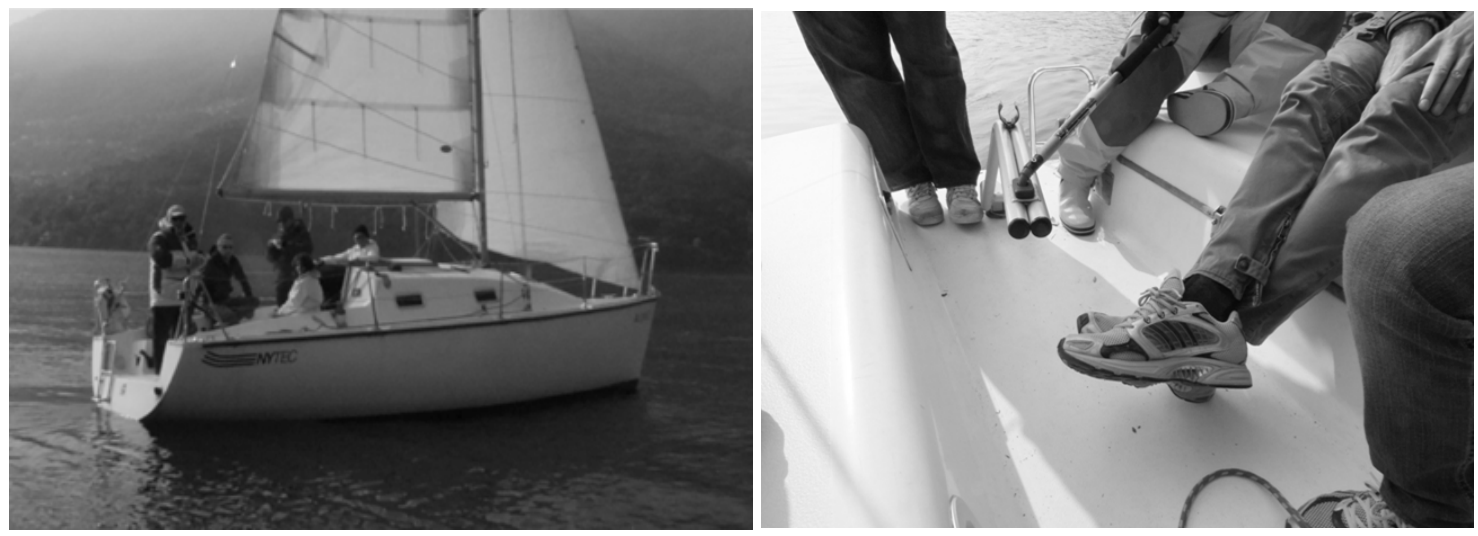

Figure 4 and 5, Lega Navale's Nytec 28 general view and boat cockpit detailed view.

\section{General Concept}

Main idea of the project is to implement a haptic control system in a pedestal winch with two handles. The new system will allow patients to trim sails. A winch pedestal will be sensorized to monitoring applied force by patient. When patient turns the handles, a central control unit will command an actuator that will trim the sails. As actuator will be used a motorized Bamar Winch (Figure 7), supplanting Lewmar winch originally mounted on Nytec 28 (figure 6). 

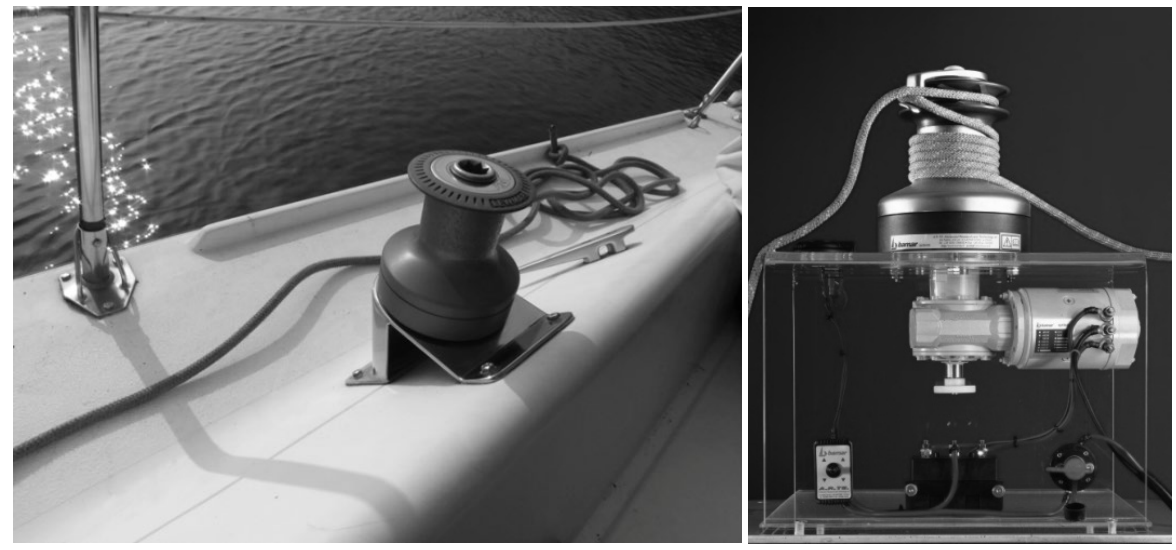

Figure 6 and 7. Lewmar original winch and Bamar motorized winch.

Handles of pedestal winch will return haptic feedback in order to allow patient to feel the applied force. At the beginning of therapy we attend an asymmetric application of force between healthy and paretic arms. These diversity will be registered by central unit and storage in order to allow medical therapist to monitoring patient evolution.

\section{Preliminary Design}

From boat measurement has been realized a 3D digital model using Rhinceros ${ }^{\circledR}$ CAD software (Figure 8). The 3D model has been useful to design a shape to apply on the boat's cockpit without modify the hull. In order to facilitate accessibility and sitting, the shape contains a handrail that creates a seat.
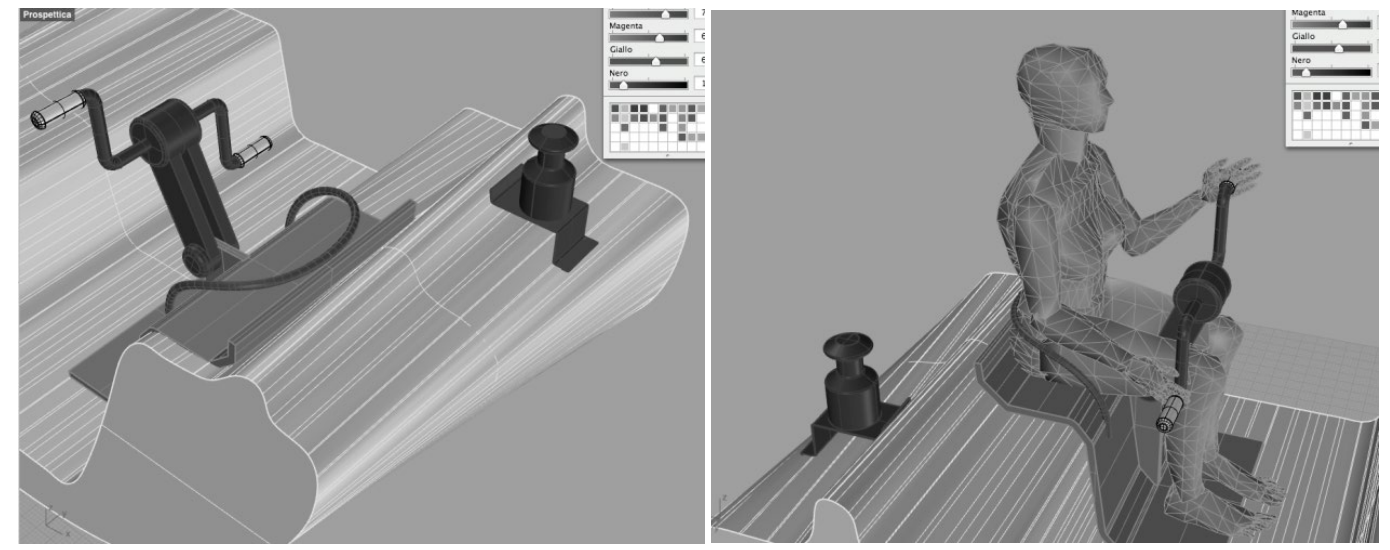

Figure 8 and 9. 3D model of cockpit with and without human model.

\section{Preliminary Virtual Evaluation}

After 3D modeling, some preliminary experiments has been made using Santos ${ }^{\circledR}$. At this stage Santos has been used only to test kinematics and no ergonomics or biomechanics evaluation has been doing. In any case, this preliminary test has been useful to determinate some possible problems related with anthropometrical variability of users and accessibility. Provably, from mechanical point of view, a removable pedestal winch will be necessary, and consequently, a wireless data transmission must be implemented. 


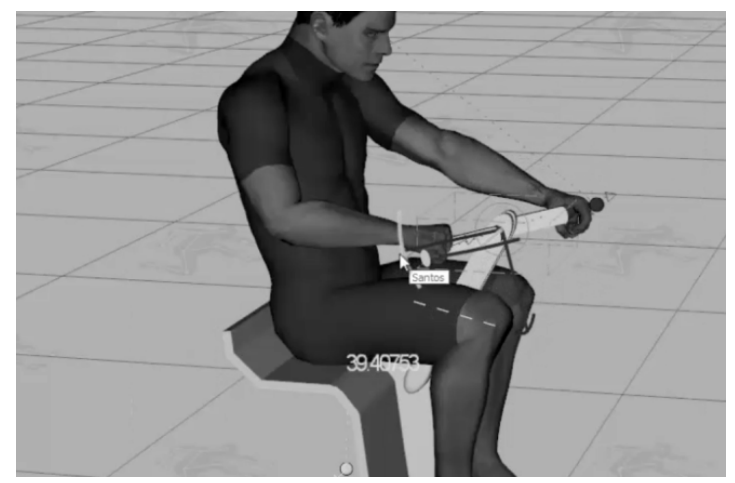

Figure 10. Screenshot of Santos preliminary test (courtesy of M. Mazzola)

\section{Physical Prototyping}

Impossibility of modifies definitively the boat hull constrain to make a reliable prototype with common navy materials. A shape of fiberglass containing a handrail, seat and handling mechanism will be produced for the second step of prototype.
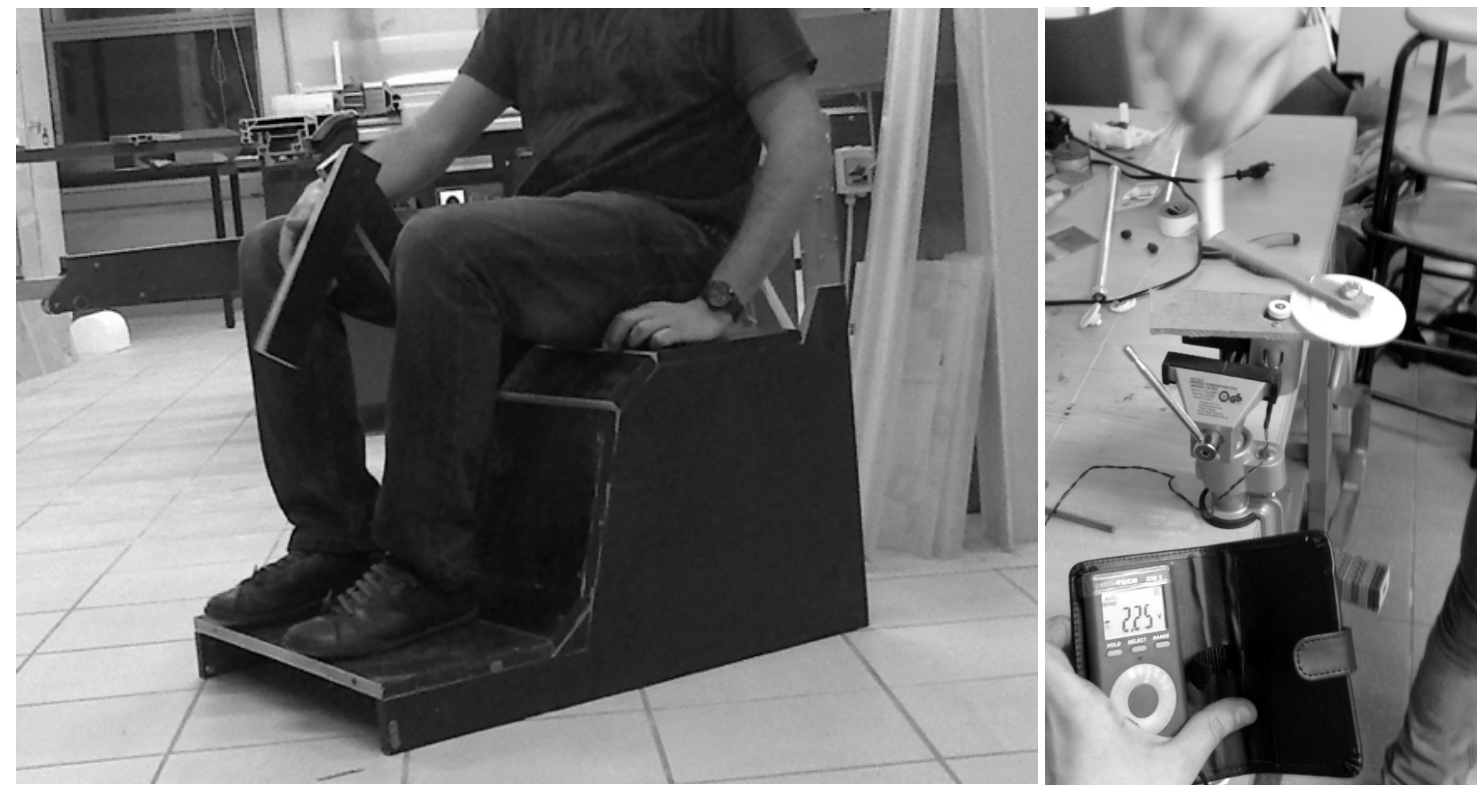

Figure 11 and 12. First prototype of cockpit and haptic winch.

At the moment, a preliminary partial prototype of cockpit has been realized to assembly the first pedestal winch (Figure 11). The prototype has been produced with wood and will be used as base to mold fiberglass shape. Contemporary, a sensorized winch has been simulated with a direct current motor in order to measure the power of a healthy arm. This preliminary test has been shown the necessity of modify approach to sensing force. Actually seams difficult to measure and actuated the resistance with the same device. And Current Controlled Hysteresis Brakes will be tested in the next step.

\section{User Test}

First user test has been organized whit Villa Beretta therapists, will involve three non critical patients which are already in Home rehab process. Will start in the Italian summer period (August), when weather in Lecco Region will be convenient. 


\section{CONCLUSIONS}

At the moment, one cycle of our research process has been completed and first problems and opportunities has been found. Prototypes has mandatory for user involvement and the trials has been rescheduled at the end of Italian spring for weather reasons. As conclusion of this step, and according with multidisciplinary team opinion, is it possible confirm utility of this project. On the other hand, working in a multidisciplinary team is as rich as complex and difficult to manage. Next step will be define technological set-up of haptic feedback and implement in a secondary cockpit prototype.

\section{ACKNOWLEDGEMENT}

The authors want to acknowledge the people how has collaborate actively in the first phase of the research. Marta Zambelli from PhyCo Lab (Laboratory of physical computing) and Marco Mazzola from LyPhe (Laboratory of Physical Ergonomics) of Design Department of Politecnico di Milano. Sailing trainers of Dervio's nautical base of Italian Navy League and occupational therapists of Villa Beretta Rehabilitation Centre from Valduce Hospital.

\section{REFERENCES}

Alankus et al. (2010), “Towards customizable games for stroke rehabilitation”. CHI 2010: Therapy and Rehabilitation, $2113-$ 2122.

Bendit Y., (2014) "RGRM wearable system”, Thesis Project, School of Design, Politecnico di Milano.

Fattore et al. (2012), "The social and economic burden of stroke survivors in Italy: a prospective, incidence-based, multi-centre cost of illness study". BMC Neurology, 12:137.

Kwakkel, G., Wagenaar, R.C., Koelman, T.W., Lankhorst, G.J., and Koetsier, J.C. (1997) "Effects of Intensity of Rehabilitation After Stroke: A Research Synthesis". Stroke 28, 8, pp. 1550-1556.

Langhorne, P., Coupar, F., and Pollock, A. (2009), “Motor recovery after stroke: a systematic review”. The Lancet Neurology 8, pp. 741-754

Langhorne et al. (2011), “Stroke Care 2, Stroke rehabilitation”. Lancet; 377: 1693-702

Lephart SM1, Pincivero DM, Giraldo JL, Fu FH., "The role of proprioception in the management and rehabilitation of athletic injuries.” Am J Sports Med. 1997 Jan-Feb;25(1):130-7.

Logan et al. (2012), "A multi-centre randomized controlled trial of rehabilitation aimed at improving outdoor mobility for people after stroke: Study protocol for a randomized controlled trial”. Trials, 13:86.

Matarić et al. (2007), "Socially assistive robotics for post-stroke rehabilitation". Journal of NeuroEngineering and Rehabilitation, 4:5 http://www.jneuroengrehab.com/content/4/1/5

Mehrholz J, Kugler J, Pohl M. (2011) "Water-based exercises for improving activities of daily living after stroke." Cochrane Database of Systematic Reviews, Issue 1. Art. No.: CD008186. DOI: 10.1002/14651858.CD008186.pub2.

NIH- National Institute of Neurological Disorders and Stroke (NINDS) (2013), "Stroke: Hope through research". www.ninds.nih.gov/disorders/stroke Accessed: 13/05/13

NSA - National Stroke Association (USA) http://www.stroke-rehab.com Accessed: 13/05/13

Piardi, S.; Ratti, A. \& Ercoli, S., (2012) "Design for All on board: Boat design in the era of access for (almost) everybody", AHFE International Conference, USA Publishing, pp. 6503-6510

Sacco S. et al. (2011), "Epidemiology of stroke in Italy". International Journal of Stroke,vol 6, pp. 219-227

Salter K., Mehta S., Bhogal S., Teasell R., Foley N., Speechley M., "Post Stroke Depression”, Cap. 18, Evidence-Based Review of Stroke Rehabilitation, $16^{\text {th }}$ edition, http://www.ebrsr.com/ Accessed: 28/02/14

Selzer, M., Clarke, S., Cohen, L., Duncan, P., and Gage, F. (2006), "Textbook of Neural Repair and Rehabilitation: Volume 2, Medical Neurorehabilitation". Cambridge Uni. Press.

Sessolo, M. (2014) "K4YOU: Kayak for stroke rehabilitation" Thesis Project, School of Design, Politecnico di Milano.

Stoller et al. (2012), "Effects of cardiovascular exercise early after stroke: systematic review and meta-analysis". BMC Neurology, 12:45

van der Lee, J.H., Wagenaar, R.C., Lankhorst, G.J., Vogelaar, T.W., Deville, W.L., and Bouter, L.M. (1999), "Forced Use of the Upper Extremity in Chronic Stroke Patients : Results From a Single-Blind Randomized Clinical Trial." Stroke 30, 11, pp. 2369-2375.

Villa, A (2014). "Atlas: Upper limb rehabilitation floating device“ Thesis Project, School of Design, Politecnico di Milano.

http://www.stroke.org/site/PageNavigator/HOME Accessed: 13/05/13 